\title{
A computational study on the influence of the delayed yielding phenomenon in magnetorheological oils on the steady state vibration and forces transmitted between the rotor and its frame
}

\author{
Jaroslav Zapoměl ${ }^{1,2,}$, Petr Ferfecki ${ }^{2,3}$, and Jan Kozánek ${ }^{1}$ \\ ${ }^{1}$ Institute of Thermomechanics, Department of Dynamics and Vibrations, Dolejškova 5, 18200 Prague, Czech Republic \\ ${ }^{2}$ VŠB - TU of Ostrava, Department of Applied Mechanics, 17. listopadu 15, 70833 Ostrava, Czech Republic \\ ${ }^{3}$ VS̆B - TU of Ostrava, IT4Innovations National Supercomputing Center, 17. listopadu 15, 70833 Ostrava, Czech Republic
}

\begin{abstract}
The theoretical analyses and practical experience show that only the damping effect adaptable to the current operating conditions makes it possible to achieve optimum performance of damping devices inserted in the supports of rotating machines. This is offered by magnetorheological squeeze film dampers. The magnetorheological oils are liquids sensitive to magnetic induction. Their response to the change of a magnetic field is not instantaneous, but it is a process called the delayed yielding. The research was focused on enhancement of the mathematical model of the magnetorheological squeeze film damper by considering the delayed yielding phenomenon and on its application for the study of the influence of the delayed yielding on the force transmission between the rotor and its stationary part. The results of the computational simulations show that rising value of the delayed yielding time constant that characterizes the delayed yielding process reduces the damping effect and efficiency of the magnetorheological damping devices.
\end{abstract}

\section{Introduction}

Unbalance is the main sources of excitation on vibration of rotors of rotating machines and of increase of forces transmitted between the rotor and its casing. A technological solution frequently used for suppressing these undesirable effects consists in inserting damping devices in the rotor supports. The analysis carried out in [1] shows that to achieve their high efficiency their damping effect must be adaptable to the operating speed. In the field of rotordynamics this is offered by squeeze film dampers lubricated by magnetorheological oils.

Wang et al. [2] used the experimental approach to study the vibration attenuation of a flexible rotor by means of a magnetorheological squeeze film damper. The design and work principles of magnetorheological dampers for rotordynamic applications are reported in [3-5]. The corresponding mathematical models applicable for computational simulations were developed and presented by Kim et al. [6], Zapoměl et al. [7-9], and other authors.

A great deal of effort of researchers was focused on the physical substance of the magnetorheological phenomenon, which consists in forming the inner structure in the flow of magnetorheological fluids [10].

The response of magnetorheological liquid materials on the change of a magnetic field is not instantaneous but it takes some time [11], which is known as the delayed yielding phenomenon. Zapoměl and Ferfecki extended mathematical models of the magnetorheological squeeze film dampers by taking this phenomenon into the consideration. [12-13] and performed the simulations.

The presented article deals with the study of the influence of the delayed yielding phenomenon on supressing amplitude of the time variable force transmitted between the rotor and its stationary part.

The principal contributions of the performed research consist in the description of the delayed yielding effect, in the development of a novel mathematical model of the magnetorheological squeeze film damper, and in learning more on behaviour of rotor systems equipped with controllable damping devices.

\section{The damping device model}

The damping effect of the magnetorheological squeeze film damper (Fig. 1) is produced by squeezing a thin layer of magnetorheological oil between two concentric rings. The magnetic flux generated in the coils embedded in the damper housing passes through the lubricant and as its resistance against the flow depends on magnetic induction, the change of the applied current can be used to change the damping force.

Magnetorheological oils are liquids composed of carrying fluid, tiny ferromagnetic particles, and of some additive materials. By application of a magnetic field the particles start to form a chain structure in the direction of the magnetic flux lines that are oriented in the damper gap in the radial direction. This effects the flow of the

Corresponding author: jaroslav.zapomel@,vsb.cz 
magnetorheological oil and is the physical substance of occurrence of the yielding shear stress.

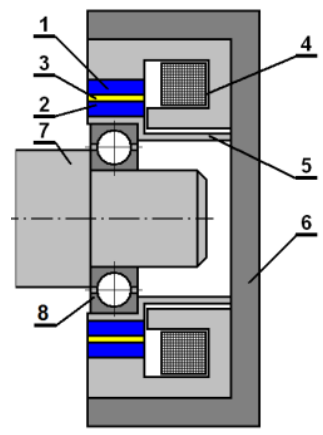

1 - outer ring

2 - inner ring

4 - electric coil

5 - cage spring

5 - cage spring
6 - damper housing

7 - shaft

8 - rolling element bearing

Fig. 1. The magnetorheological squeeze film damper.

The developed mathematical model of the magnetorheological damper is based on assumptions of the classical theory of lubrication $[14,15]$, only the magnetorheological oil is represented by bilinear material. In addition, it is assumed that the geometric and design parameters allow to consider the damper as short $[14,15]$.

Then the pressure distribution in the full oil film is governed by the modified Reynolds equation [9]

$$
\begin{gathered}
\frac{\partial}{\partial Z}\left(\frac{1}{\eta_{C}} h^{3} p^{\prime}\right)=12 \dot{h} \\
\frac{\partial}{\partial Z}\left[\frac{1}{\eta}\left(h^{3} p^{\prime}+3 h^{2} \tau_{y}+8 \frac{\tau_{C}^{3}}{p^{\prime 2}}-12 \frac{\tau_{y} \tau_{C}^{2}}{p^{\prime 2}}\right)-\frac{8}{\eta_{C}} \frac{\tau_{C}^{3}}{p^{\prime 2}}\right]=12 \dot{h} \\
Z_{C}=-\frac{\tau_{C} h^{2}}{6 \eta_{C} \dot{h}} \\
p_{C}^{\prime}=-\frac{2 \tau_{C}}{h}
\end{gathered}
$$

$p$ is the pressure, $p^{\prime}$ stands for the pressure gradient in the axial direction, $h$ is the oil film thickness, $Z$ is the axial coordinate defining position in the oil film, $\tau_{y}$ is the yielding shear stress, $\tau_{C}$ is the shear stress at the core border of bilinear materials, and $\eta_{C}, \eta$ are the dynamic viscosities of the oil in and outside of the core region, respectively, $p_{C}^{\prime}$ denotes the pressure gradient in the axial direction at location given by the axial coordinate $Z_{C}$, and (') reads for the first derivative with respect to time.

A cavitation is assumed in those parts of the damper gap where the thickness oil the film rises with time. In cavitated areas the pressure of the medium remains constant and equal to the pressure in the ambient space.

The damper housing is considered to be composed of a set of meridian segments and each segment as a divided core of an electromagnet with the gap filled with the magnetorheological oil. This makes it possible to express magnetic induction $B$ in the oil film

$$
B=k_{B} \mu_{0} \mu_{r} \frac{I}{h}
$$

$k_{B}$ is the damper design parameter [16], $\mu_{0}$ is the vacuum permeability, $\mu_{r}$ is the relative permeability of the magnetorheological oil, and $I$ is the applied current.

The dependence of the stationary value of the yielding shear stress on magnetic induction can be approximated by a power function

$$
\tau_{y}=k_{y} B^{n_{y}}
$$

$k_{y}$ and $n_{y}$ are the material constants of the magnetorheological oil.

The components of the magnetorheological damping force acting on the rotor journal are given by integration of the oil pressure distribution around the damper circumference and along its length.

The physical substance of the yielding shear stress of magnetorheological fluids is a chain structure of ferromagnetic particles dispersed in the carrying liquid if a magnetic field is applied. Its forming or changing is a process that takes some time. It implies the yield shear stress depends not only on instantaneous magnitude of the magnetic induction but also on its history in the past. This is well described by a convolutory integral. Utilizing the exponential kernel and relation (6) the time history of the yielding shear stress accounting for the delayed yielding phenomenon reads

$$
\tau_{y}=\frac{k_{y}}{T_{y}} \int_{0}^{t} e^{-\frac{1}{T_{y}(t-\vartheta)} B^{n_{y}}} \mathrm{~d} \vartheta
$$

$T_{y}$ is the delayed yielding time constant characterizing rapidity of the process of forming the particle structure inside the magnetorheological oil layer and $\vartheta$ denotes the time in the past before the moment of time $t$.

Consequently, relation (7) is transformed to the differential form

$$
T_{y} \dot{\tau}_{y}+\tau_{y}=k_{y} B^{n_{y}}
$$

In areas, in which the cavitation takes place, it is assumed that the particle structure is completely disrupted and as a result, the yielding shear stress is considered to become zero there.

\section{The investigated rotor system}

The developed novel mathematical model of the magnetorheological squeeze film damper was applied for investigation of influence of the delayed yielding phenomenon on vibration attenuation and on the force transmission between the rotor and its stationary part during the steady state operating regime.

The studied rotor (Fig. 2) is rigid. At both its ends it is coupled with a rigid casing by two magnetorheological squeeze film dampers. The rotor rotates at constant angular speed and is loaded by its weight and by the disc unbalance. The deflection of the cage springs caused by the rotor weight is eliminated by their prestressing. The whole system can be considered as symmetric with respect to the middle plane of the disc.

The dampers are implemented in the computational model by springs and force couplings. 


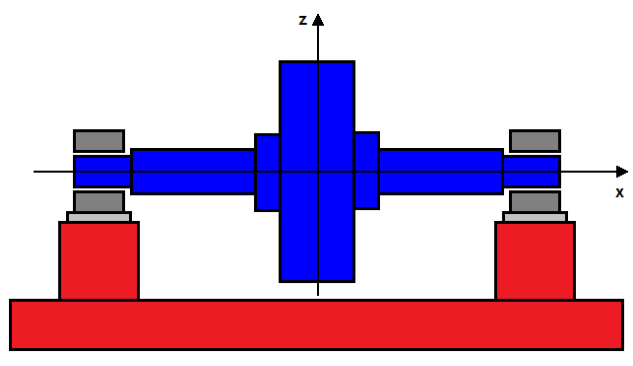

Fig. 2. The investigated rotor system.

Because of the system symmetry the lateral vibration of the rotor is governed by a set of two nonlinear motion equations

$$
\begin{aligned}
m_{R} \ddot{y}+b_{P} \dot{y}+2 k_{D} y & =2 F_{m y}+m_{R} e_{T} \omega^{2} \cos \left(\omega t+\psi_{o}\right) \\
m_{R} \ddot{z}+b_{P} \dot{z}+2 k_{D} z & =2 F_{m r z}+m_{R} e_{T} \omega^{2} \sin \left(\omega t+\psi_{o}\right) \\
& -m_{R} g+2 F_{P S}
\end{aligned}
$$

$m_{R}$ is the rotor mass, $b_{P}$ is the coefficient of the rotor external damping, $k_{D}$ is the bending stiffness of one cage spring, $e_{T}$ is the eccentricity of the rotor centre of gravity, $F_{m r y}, F_{m r z}$ are the $\mathrm{y}$ and $\mathrm{z}$ components of the magnetorheological damping force acting on the rotor journal, $F_{P S}$ is the prestressing force, $\omega$ is the angular speed of the rotor rotation, $\psi_{o}$ is the phase shift, $g$ is the gravity acceleration, $y, z$ are the displacements of the rotor centre in the horizontal and vertical directions, and (*) notates the second derivative with respect to time.

\section{Results of the simulations}

The technological and operating parameters of the system are: the rotor mass $450 \mathrm{~kg}$, the bending stiffness of each damper cage spring $5.0 \mathrm{MN} / \mathrm{m}$, the viscous damping coefficient (external damping) $10 \mathrm{Nm} / \mathrm{s}$, the disc unbalance $45 \mathrm{kgmm}$, the oil viscosity (if not affected by a magnetic field) 0.3 Pas, the damper gap mean diameter $150 \mathrm{~mm}$, the damper length $50 \mathrm{~mm}$, the damper clearance $1.0 \mathrm{~mm}$, the damper design parameter 60 , the magnetorheological oil relative permeability 5 , and the proportional and exponential constants $10000 \mathrm{PaT}^{-1.1}, 1.1$. The applied current is $0.2 \mathrm{~A}$.

A simple dynamical analysis shows that the critical speed is $149.1 \mathrm{rad} / \mathrm{s}$

Fig. 3 and 4 are related to the rotor angular speed of $100 \mathrm{rad} / \mathrm{s}$, which is lower than the critical one. The figures show the steady state orbit of the rotor centre and the time history of the force transmitted between the rotor and the stationary part in the horizontal direction. The cage springs of both magnetorheological dampers are prestressed, which results in a circular shape of the orbit. It is evident that the application of a fluid with higher delayed yielding time constant arrives at increase of the size of the orbit and amplitude of the transmitted force (Fig. 4).

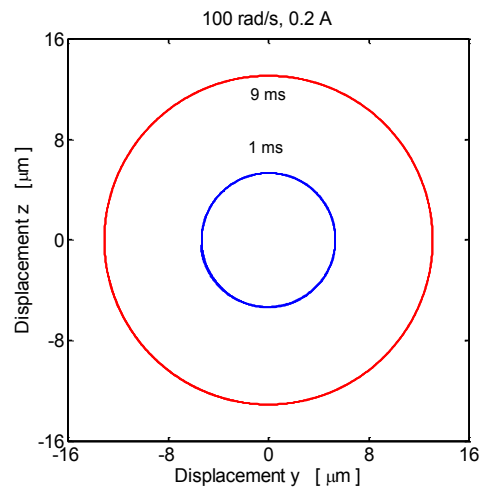

Fig. 3. Orbit of the rotor centre (100 rad/s).

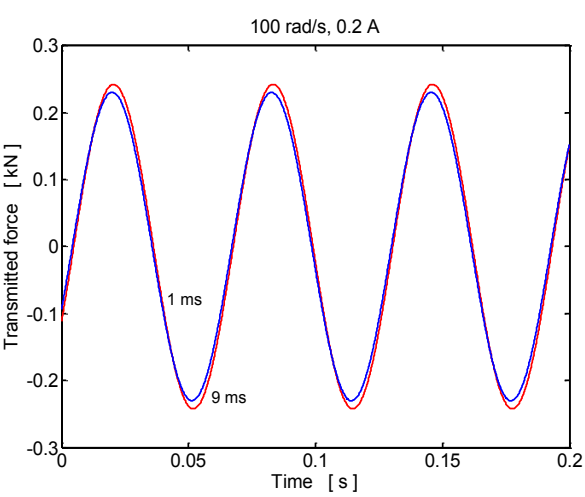

Fig. 4. Transmitted force $(100 \mathrm{rad} / \mathrm{s})$.

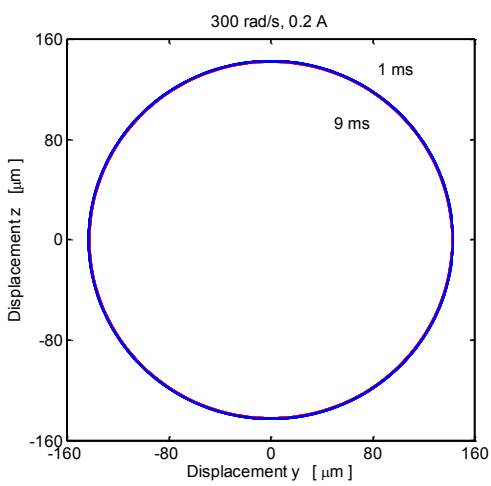

Fig. 5. Orbit of the rotor centre (300 rad/s).

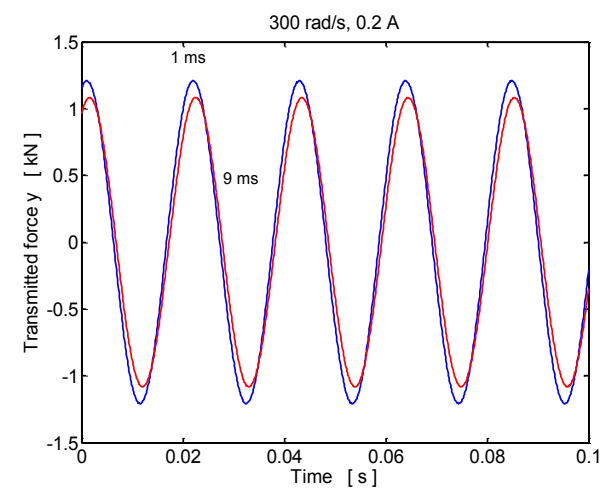

Fig. 6. Transmitted force $(300 \mathrm{rad} / \mathrm{s})$.

The orbit and the time history of the force transmitted to the rotor casing drawn in Fig. 5 and 6 are related to the operating regime when the rotor turns at angular 
speed of $300 \mathrm{rad} / \mathrm{s}$, which is higher than the critical velocity. The results show that magnitude of the delayed yielding time constant has almost no influence on the orbit size but its lower value leads to increase of the transmitted force.

Fig. 7 and 8 show the time history of the yielding shear stress at a specified location in the damper gap for two magnitudes of the delayed yielding time constant. The rotor turns at angular speed of $300 \mathrm{rad} / \mathrm{s}$. As evident, lower value of the time constant arrives at steeper increase of the yielding shear stress and at reaching its higher magnitude. During the periods when a cavitation takes place the yielding shear stress falls to zero.

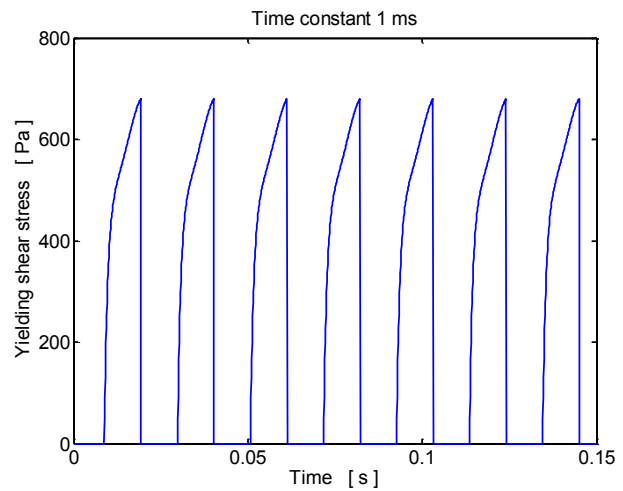

Fig. 7. Time history of the yielding shear stress $(1 \mathrm{~ms})$.

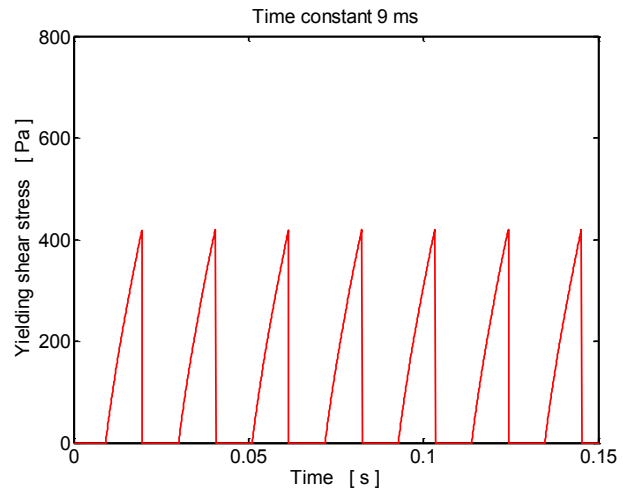

Fig. 8. Time history of the yielding shear stress $(9 \mathrm{~ms})$.

\section{Conclusions}

The research was aimed at investigation of the influence of the delayed yielding phenomenon on reducing the force transmitted from the rotor to its stationary part.

The results of the computational simulations show that lower value of the delayed yielding time constant leads to a smaller orbit and lower amplitude of the transmitted force for the velocity lower than the critical speed. For the velocity higher than the critical one lower value of the time constant increases the maximum transmitted force and has almost no influence of the orbit size. Such behaviour of the rotor shows that rising value of the delayed yielding time constant reduces the damping effect and efficiency of the damping device.

A more accurate description of behaviour of magnetorheological oils, the enhancement of the mathematical model of a short squeeze film damper by taking into consideration the delayed yielding phenomenon, and learning more on effect of magnetorheological dampers on oscillation of rotating machines are the main contributions of the carried out research.

The research work reported in this paper was made possible by the Czech Science Foundation (project no. 15-06621S) and the national Programme of Sustainability (project LQ1602 IT4Innovations excellence in science). The support is highly acknowledged.

\section{References}

1. J. Zapoměl, P. Ferfecki, J. Kozánek, Appl. Comput. Mech., 7 (2013)

2. J. Wang, G. Meng, E. J. Hahn, Proceedings of the 2003 ASME Design Engineering Technical Conference \& Computers and Information in Engineering Conference, 955-959 (2003)

3. J. Wang, G. Meng, Proc. Inst. Mech. Eng. Part L J. Mater. Des. Appl., 215 (2001)

4. X. Gong, X. Ruan, S. Xuan, Q. Yan, H. Deng, Adv. Mech. Eng., 6 (2014)

5. P. Forte, M. Paterno, E. Rustighi, Int. J. Rotat. Mach., 10 (2004)

6. K. J. Kim, C. W. Lee, J. H. Koo, Smart Mater. Struct., 17 (2008)

7. J. Zapoměl, P. Ferfecki, P. Forte, Smart Mater. Struct., 21 (2012)

8. J. Zapoměl, P. Ferfecki, Model. Opt. Phys. Syst., 9 (2010)

9. J. Zapoměl, P. Ferfecki, Appl. Math. Model., 52 (2017)

10. R. Tao, J. Phys. Condens. Matter, 13 (2001)

11. H. Li, X. Peng, W. Chen, J. Intell. Material Syst. Struct., 16 (2005)

12. J. Zapoměl, P. Ferfecki, Proceedings of the $9^{\text {th }}$ IFToMM International Conference on Rotor Dynamics, 1021-1032 (2014)

13. J. Zapoměl, P. Ferfecki, Proceedings of the $5^{\text {th }}$ ECCOMAS Thematic Conference on Computational Methods in Structural Dynamics and Earthquake Engineering, 1941-1948 (2015)

14. J. Zapoměl, Computer Modelling of Lateral Vibration of Rotors Supported by Hydrodynamical Bearings and Squeeze Film Dampers, VSBTechnical University of Ostrava, (2007, in Czech)

15. A. Z. Szeri, Tribology: Friction, Lubrication, and Wear, (1980)

16. P. Ferfecki, J. Zapoměl, J. Kozánek, Adv. Eng. Soft., 104 (2017) 